\title{
Effects of Chemical Reaction on Dissipative Radiative MHD Flow through a Porous Medium over a Nonisothermal Stretching Sheet
}

\author{
S. Mohammed Ibrahim \\ Department of Mathematics, Priyadarshini College of Engineering \& Technology, Nellore, Andhra Pradesh 524004, India \\ Correspondence should be addressed to S. Mohammed Ibrahim; ibrahimsvu@gmail.com
}

Received 25 August 2013; Revised 22 February 2014; Accepted 5 March 2014; Published 1 April 2014

Academic Editor: Santiago Laín

Copyright (C) 2014 S. Mohammed Ibrahim. This is an open access article distributed under the Creative Commons Attribution License, which permits unrestricted use, distribution, and reproduction in any medium, provided the original work is properly cited.

\begin{abstract}
The steady two-dimensional radiative MHD boundary layer flow of an incompressible, viscous, electrically conducting fluid caused by a nonisothermal linearly stretching sheet placed at the bottom of fluid saturated porous medium in the presence of viscous dissipation and chemical reaction is studied. The governing system of partial differential equations is converted to ordinary differential equations by using the similarity transformations, which are then solved by shooting method. The dimensionless velocity, temperature, and concentration are computed for different thermophysical parameters, namely, the magnetic parameter, permeability parameter, radiation parameter, wall temperature parameter, Prandtl number, Eckert number, Schmidt number, and chemical reaction.
\end{abstract}

\section{Introduction}

The fluid flows with chemical reaction have attracted the attention of engineers and scientists in the recent times. Such flows have key importance in many processes including drying evaporation at the surface of a water body, energy transfer in a wet cooling tower, flow in a desert cooler, generating electric power, food processing, groves of fruit trees, and crops damage because of freezing. There is always a molecular diffusion of species in the presence of chemical reaction within or at the boundary during several practical diffusive operations. There are two types of reactions, namely, homogeneous and heterogeneous. A homogeneous reaction takes place uniformly in the entire given phase whereas a heterogeneous reaction exists in a restricted region or within the boundary of a phase. The smog formation is an important example representing a first-order homogeneous chemical reaction. Several researchers in view of such facts are engaged in the discussion of flows with chemical reactions. Forinstance Seddeek and Almushigeh [1] investigated the effects of radiation and variable viscosity on MHD free convective flow and mass transfer over a stretching sheet with chemical reaction. Kandasamy et al. [2] presented group analysis for Soret and Dufour effects on free convective heat and mass transfer with thermophoresis and chemical reaction over a porous stretching surface in the presence of heat source/sink. $\mathrm{Pal}$ and Talukdar [3] presented the combined effects of Joule heating and chemical reaction on unsteady magnetohydrodynamic mixed convection with viscous dissipation over a vertical plate in the presence of porous media and thermal radiation. Joneidi et al. [4] presented analytical treatment of MHD free convection flow over a stretching sheet with chemical reaction. Anjalidevi and Kandasamy [5] studied effects of chemical reaction, heat and mass transfer on laminar flow along a semi-infinite horizontal plate. There are intensive studies which have been carried out to investigate effects of chemical reaction on different flow types (see Seddeek et al. [6]; Salem and Abd El-Aziz [7]).

The study of convection heat and mass transfer phenomenon in porous media has gained attention due to its interesting applications. Processes involving heat and mass transfer in porous media are often encountered in the chemical industry, in reservoir engineering in connection with thermal recovery process, and in the study of dynamics of hot 
and salty springs of a sea. Underground spreading of chemical wastes and other pollutants, grain storage, evaporation cooling, and solidification are the few other application areas where the combined thermosolutal convection in porous media is observed. Detailed reviews of flow through and past porous media can be found in Nield and Bejan [8]. Abel et al. [9] studied the two-dimensional boundary layer problem on mixed convection of an incompressible viscoelastic fluid immersed in porous medium over a stretching sheet. Vyas and Srivastava [10] investigated the radiation effect on MHD flow over a nonisothermal stretching sheet in a porous medium.

Magnetohydrodynamic (MHD) boundary layers with heat and mass transfer over nonisothermal stretching sheet are found in many engineering and geophysical applications such as geothermal reservoirs, thermal insulation, enhanced oil recovery, packed-bed catalytic reactors, and cooling of nuclear reactors. Many chemical engineering processes like metallurgical and polymer extrusion processes involve cooling of a molten liquid being stretched into a cooling system. The use of magnetic field that influences heat generation/absorption process in electrically conducting fluid flows has many engineering applications, for example, many metallurgical processes which involve cooling of continuous strips or filaments, which are drawn through a quiescent fluid. The properties of the final product depend to a great extent on the rate of cooling. The rate of cooling and, therefore, the desired properties of the end product can be controlled by the use of electrically conducting fluids and the applications of the magnetic fields (Vajravelu and Hadjinicolaou [11]). Many works have been reported on flow, heat and mass transfer of electrically conducting fluids over semi-infinite/infinite plates/stretching surfaces in the presence of magnetic field (see, e.g., Mohammed Ibrahim and Bhaskar Reddy [12]; Shateyi et al. [13]; Makinde and Sibanda [14]; Pal and Talukdar [15]; and Makinde and Aziz [16]).

Radiative heat and mass transfer play an important role in manufacturing industries for the design of fins, steel rolling, nuclear power plants, and gas turbines and various propulsion devices for aircraft, missiles, satellites, and space vehicles are examples of such engineering applications. The effect of radiation on MHD flow, heat and mass transfer becomes more important industrially. Many processes in engineering areas occur at high temperature and knowledge of radiation heat transfer becomes very important for the design of the pertinent equipment. The quality of the final product depends to a great extent on the heat controlling factors, and the knowledge of radiative heat transfer in the system can lead to a desired product with sought qualities. Different researches have been forwarded to analyze the effects of thermal radiation on different flows (Cortell [17]; Ibrahim et al. [18]; Shateyi [19]; Shateyi and Motsa [20]; and Aliakbar et al. [21]).

Viscous mechanical dissipation effects are important in geophysical flows and also in certain industrial operations and are usually characterized by the Eckert number. Gebhart [22] came out with observations that devices which operate at high rotational speeds or which are subject to large decelerations experience significant viscous dissipation effect. The



Figure 1: Physical model.

effect is felt prominently in strong gravitational fields and in processes wherein the scale of the process is very large, for example, on larger planets, in large masses of gas in space, and in geological processes in fluids contained in various bodies. It is pertinent to record that even if viscous dissipation effect is quantitatively negligible in some cases, its qualitative effect is significantly observed. Vyas and Srivastava [23] studied the radiation effects on MHD boundary layer flow in a porous medium over a nonisothermal stretching sheet in presence of dissipation. The works of Gebhart and Mollendorf [24], Nield [25], and Rees et al. [26] shed light on the importance of viscous heating.

The objective of this paper was to explore the effects of thermal radiation, viscous dissipation, and chemical reaction on MHD boundary layer flow over a nonisothermal stretching sheet embedded in porous medium. Using a similarity approach, the governing equations are transformed into nonlinear ordinary equations and solved numerically using shooting technique along with fourth-order Runge-Kutta method. The pertinent results are displayed graphically and discussed quantitatively.

\section{Mathematical Model}

Consider the steady two-dimensional forced convection boundary-layer flow of viscous, incompressible, electrically conducting fluid in a fluid saturated horizontal porous medium caused by linearly stretching sheet placed at the bottom of the porous medium. A Cartesian coordinate system is used. The $x$-axis is along the sheet and $y$-axis is normal to the $x$-axis (see Figure 1 ). Two equal and opposite forces are applied along the sheet so that the wall is stretched, keeping the position of the origin unaltered. The stretching velocity varies linearly with the distance from the origin. A uniform magnetic field of strength $B_{0}$ is applied normal to sheet. We assume that the wall temperature $T_{w}>T_{\infty}$, where $T_{\infty}$ is the uniform temperature of the ambient temperature. We also assume that the fluid is optically dense, Newtonian, and without phase change. Further it is assumed that both the fluid and the porous medium are in local thermal 
equilibrium. We also consider that both the surroundings and the fluid are maintained at a constant temperature $T_{\infty}$ far away from the sheet. We further assume that there exists a homogeneous chemical reaction between the fluid and species concentration. The foreign mass present in the flow is assumed to be at low level and hence Soret and Dufour effects are negligible.

Under these assumptions and considering the viscous dissipation, the governing boundary layer equations for the momentum, heat and mass transfer in the presence of thermal radiation, and chemical reaction take the following form of the governing equations is given by:

$$
\begin{gathered}
\frac{\partial u}{\partial x}+\frac{\partial u}{\partial y}=0 \\
u \frac{\partial u}{\partial x}+v \frac{\partial u}{\partial y}=v \frac{\partial^{2} u}{\partial y^{2}}+g \beta\left(T-T_{\infty}\right)+g \beta^{*}\left(C-C_{\infty}\right) \\
-\frac{\sigma B_{0}^{2}}{\rho} u-\frac{v}{k^{\prime}} u, \\
u \frac{\partial T}{\partial x}+v \frac{\partial T}{\partial y}=\frac{k}{\rho c_{p}} \frac{\partial^{2} T}{\partial y^{2}}-\frac{1}{\rho c_{p}} \frac{\partial q_{r}}{\partial y}+\frac{\mu}{\rho c_{p}}\left(\frac{\partial u}{\partial y}\right)^{2} \\
u \frac{\partial C}{\partial x}+v \frac{\partial C}{\partial y}=D \frac{\partial^{2} C}{\partial y^{2}}-K^{*}\left(C-C_{\infty}\right) .
\end{gathered}
$$

The boundary conditions for the velocity, temperature, and concentration fields are

$$
\begin{aligned}
& u=c x, \quad v=0, \quad T=T_{w}=T_{\infty}+d x^{\alpha}, \\
& C=C_{w}=C_{\infty}+d x^{\alpha} \text { at } y=0 \\
& u \longrightarrow 0, \quad T \longrightarrow T_{\infty}, \quad C \longrightarrow C_{\infty} \quad \text { as } y \longrightarrow \infty \text {, }
\end{aligned}
$$

where $x$ and $y$ represent the coordinate axes along the continuous stretching surface in the direction of motion and normal to it, respectively, $u$ and $v$ are the velocity components along the $x$-and $y$-axes respectively, $v$ is the kinematics viscosity, $\mu$ is the fluid viscosity, $\sigma$ is electric conductivity, $B_{0}$ is the uniform magnetic field, $\rho$ is the density, $k^{\prime}$ is the permeability of the porous medium, $c_{p}$ is the specific heat at constant pressure, $q_{r}$ is the radiation heat flux, $T$ is the temperature inside the boundary layer, $T_{\infty}$ is the temperature far away from the plate, $C$ is the species concentration in the boundary layer, $C_{\infty}$ is the concentration far away from the plate, $\beta$ is the coefficient of thermal expansion, $\beta^{*}$ is the coefficient of concentration expansion, $g$ is the gravitational acceleration, $\alpha$ is the wall temperature parameter, $D$ is the molecular diffusivity of the species concentration, $c>0, d$ are constants, and $\mathrm{Kr}^{*}$ is the first-order homogeneous constant reaction rate.

The radiative heat flux $q_{r}$ is described by Roseland approximation for radiation (Brewster [27]) such that

$$
q_{r}=-\frac{4 \sigma^{*}}{3 K^{*}} \frac{\partial T^{4}}{\partial y},
$$

where $\sigma^{*}$ and $K^{*}$ are Stefan-Boltzmann constant and mean absorption coefficient, respectively. Following Chamkha [28], we assume that the temperature differences within the flow are sufficiently small so that $T^{4}$ can be expressed as a linear function after using Taylor series to expand $T^{4}$ over the free stream temperature $T_{\infty}$ and neglecting higher-order terms. This result is the following approximation:

$$
T^{4} \approx 4 T_{\infty}^{3} T-3 T_{\infty}^{4} .
$$

Using (6) and (7) in (3), we obtain

$$
\frac{\partial q_{r}}{\partial y}=-\frac{16 \sigma^{*}}{3 K} \frac{\partial^{2} T^{4}}{\partial y^{2}} .
$$

We introduce the following nondimensional variables:

$$
\begin{aligned}
& \eta=\sqrt{\frac{\bar{c}}{v}} y, \quad u=\frac{\partial \psi}{\partial y}=x c f^{\prime}, \\
& v=-\frac{\partial \psi}{\partial x}=-\sqrt{c \nu} f \\
& M=\frac{\sigma B_{0}^{2}}{\rho c}, \quad K=\frac{v}{k^{\prime} c} \\
& \theta=\frac{T-T_{\infty}}{T_{w}-T_{\infty}}, \quad \phi=\frac{C-C_{\infty}}{C_{w}-C_{\infty}}, \\
& R=\frac{K k^{*}}{4 \sigma T_{\infty}^{3}}, \\
& \operatorname{Pr}=\frac{\rho \nu c_{p}}{k}, \quad \mathrm{Gr}=\frac{g \beta\left(T_{w}-T_{\infty}\right)}{c^{2} x} \\
& \mathrm{Gc}=\frac{g \beta^{*}\left(C_{w}-C_{\infty}\right)}{c^{2} x}, \\
& \mathrm{Ec}=\frac{c^{2} x^{2}}{c_{p}\left(T_{w}-T_{\infty}\right)}, \\
& \mathrm{Sc}=\frac{\nu}{D}, \quad \mathrm{Kr}=\frac{\mathrm{Kr}^{*}}{c} .
\end{aligned}
$$

In view of (6), (2)-(4) take the form:

$$
\begin{gathered}
f^{\prime \prime \prime}+f f^{\prime \prime}-\left(f^{\prime}\right)^{2}+\operatorname{Gr} \theta+\operatorname{Gc} \phi-(M+K) f^{\prime}=0, \\
\theta^{\prime \prime}-\left(\frac{3 \operatorname{Pr} R}{3 R+4}\right)\left[\alpha \theta f^{\prime}-f \theta^{\prime}\right]+\left(\frac{3 \operatorname{Pr} R}{3 R+4}\right) \operatorname{Ec}\left(f^{\prime \prime}\right)^{2}=0, \\
\phi^{\prime \prime}+\operatorname{Sc} f \phi^{\prime}-\operatorname{Kr} \operatorname{Sc} \phi=0,
\end{gathered}
$$

where the primes denote the differentiation with respect to $\eta, M$ is the magnetic parameter, $K$ is the permeability parameter, $\mathrm{Pr}$ is the Prandtl number, $R$ is the radiation parameter, $\alpha$ is wall temperature parameter, Gr is the Grashof number, Gc is the modified Grashof number, Ec is the Eckert number, Sc is the Schmidt number, and $\mathrm{Kr}$ is the chemical reaction parameter. 
The corresponding boundary conditions are

$$
\begin{gathered}
f^{\prime}=1, \quad f=0, \quad \theta=1, \quad \phi=1 \quad \text { at } \eta=0 \\
f^{\prime}=0, \quad \theta=0, \quad \phi=0 \quad \text { as } \eta \longrightarrow \infty .
\end{gathered}
$$

\section{Numerical Computation}

The numerical solutions of the nonlinear differential equations (10) under the boundary conditions (11) have been performed by applying a shooting method along with the fourth-order Runge-Kutta method. First of all higher-order nonlinear differential equations (10) are converted into simultaneous linear differential equations of first order and they are further transformed into initial value problem by applying the shooting technique. From this process of numerical computation, the skin-friction coefficient, the Nusselt number, and Sherwood number which are, respectively, proportional to $f^{\prime \prime}(0),-\theta^{\prime}(0)$, and $-\phi^{\prime}(0)$ are also sorted out and their numerical values are presented in a tabular form.

\section{Results and Discussion}

To analyze the results, numerical computation has been carried out using the method described in the previous paragraph for various governing parameters, namely, thermal Grashof number Gr, modified Grashof number Gc, the magnetic field parameter $M$, permeability parameter $K$, Prandtl number Pr, wall temperature parameter $\alpha$, thermal radiation parameter $R$, Eckert number Ec, Schmidt number Sc,and chemical reaction parameter $\mathrm{Kr}$. In the present study the following default parameter values are adopted for computations: $\mathrm{Gr}=2.0, \mathrm{Gc}=2.0, M=1.0, K=0.5, \mathrm{Pr}=$ $0.72, R=1.0, \alpha=1.0, \mathrm{Ec}=0.5, \mathrm{Sc}=0.6$, and $\mathrm{Kr}=$ 0.5. All graphs therefore correspond to these values unless specifically indicated on the appropriate graph.

The influence of the thermal Grashof number $\mathrm{Gr}$ on the velocity is presented in Figure 2. It is observed that there is a rise in the velocity due to enhancement of thermal buoyancy force. Figure 3 presents typical velocity profiles in the boundary layer for various values of the solutal Grashof number Gc, while all other parameters are kept at some fixed values. As expected, the fluid velocity increases and the peak value is more distinctive due to increase in the species buoyancy force.

The effects of the magnetic field parameter $M$ on the dimensionless velocity, temperature, and concentration fields are shown in Figures 4, 5, and 6; it is obvious that an increase in the magnetic field parameter $M$ results in a decrease in the velocity. It is observed that the temperature and concentration profiles increase with increasing of magnetic field parameter $M$.

Figures 7, 8, and 9 show the dimensionless velocity, temperature, and concentration profiles for different values of permeability parameter $K$. It can be seen that the velocity profile decreases with the increase of permeability parameter $K$. It is noticed that the temperature and concentration profiles increase permeability parameter $K$.

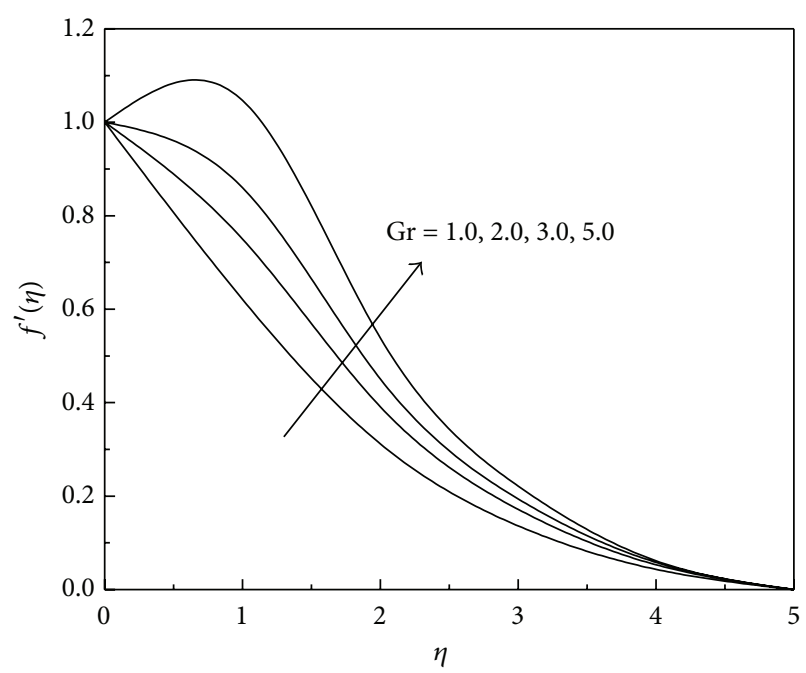

FIGURE 2: Velocity profiles for different values of Gr.

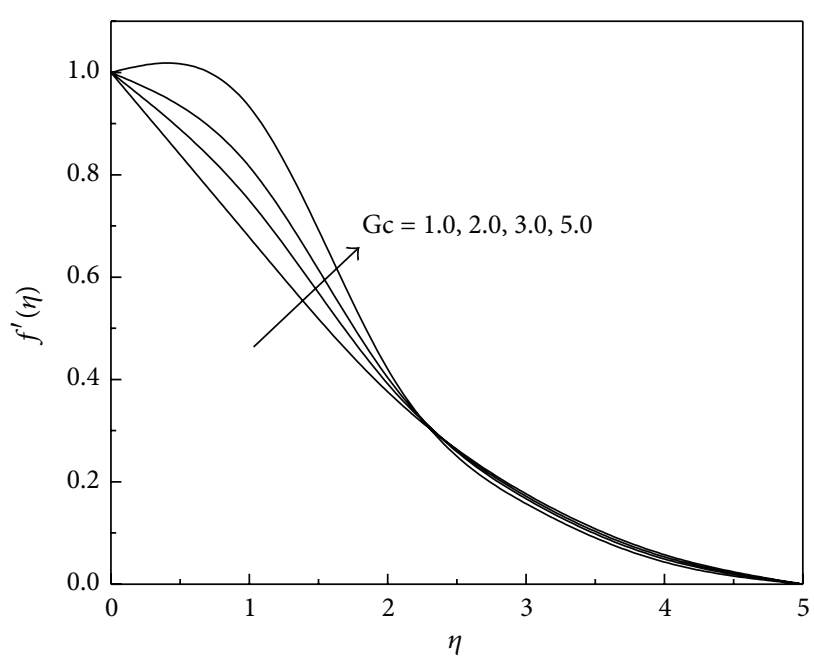

FIGURE 3: Velocity profiles for different values of Gc.

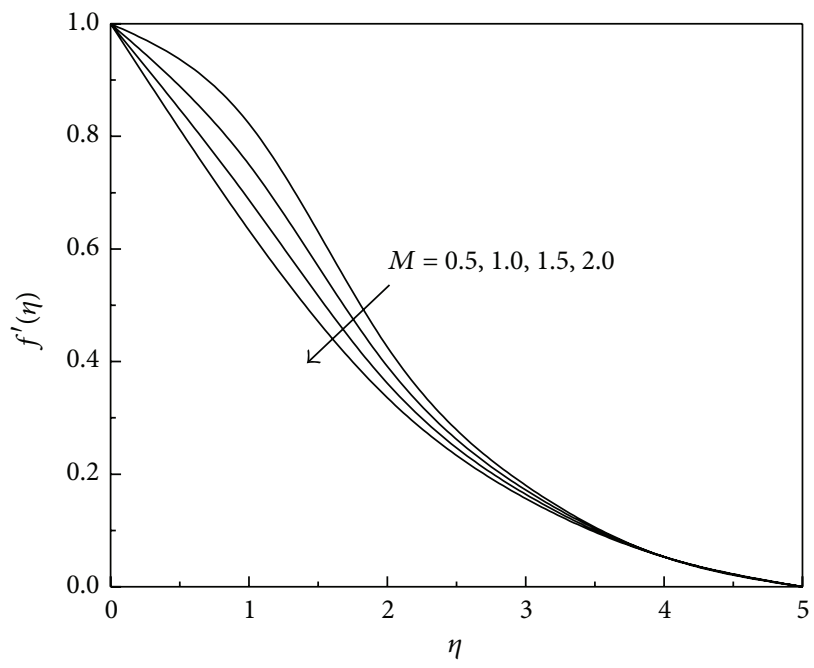

FIgURE 4: Velocity profiles for different values of $M$. 


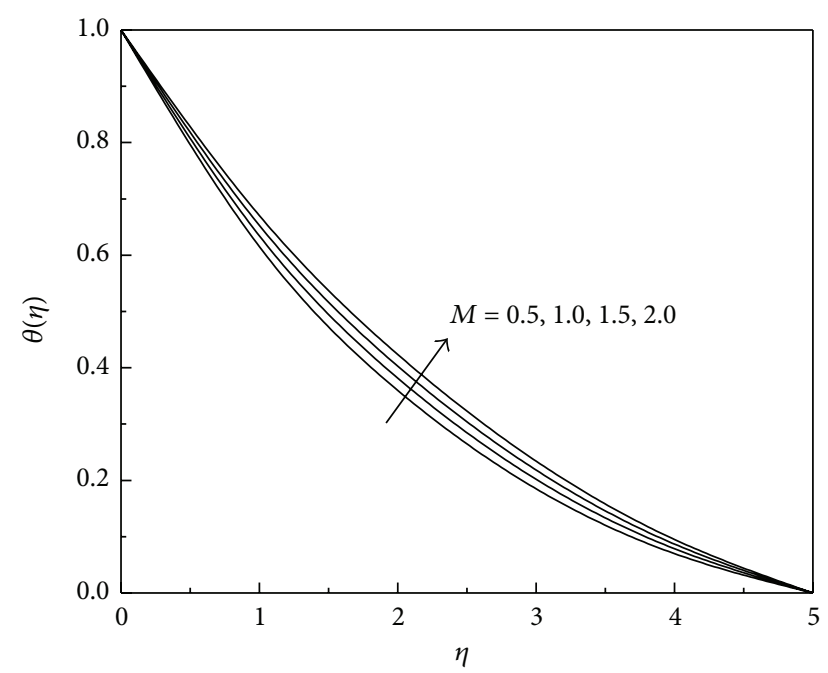

FIgURE 5: Temperature profiles for different values of $M$.



FiguRE 6: Concentration profiles for different values of $M$.

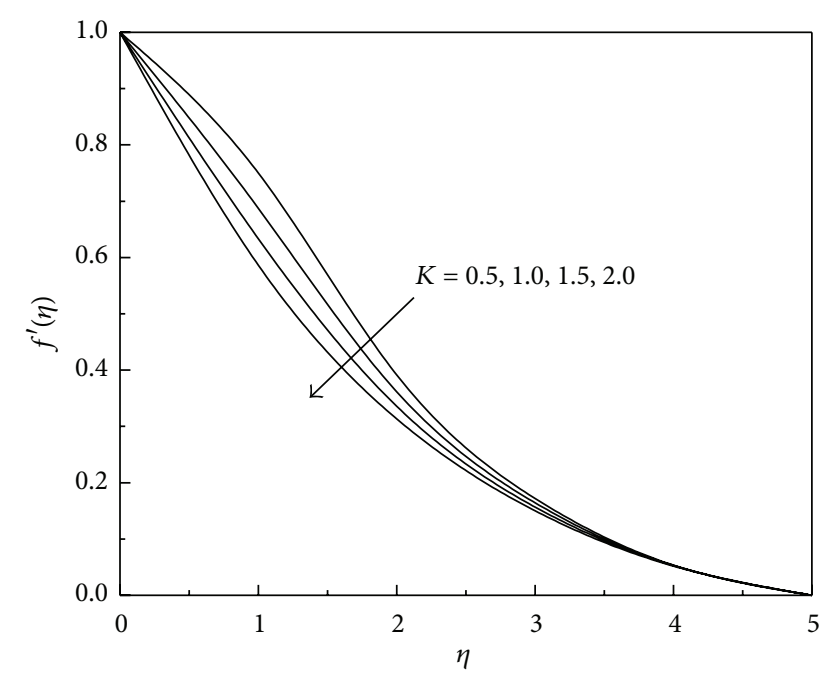

FIgURE 7: Velocity profiles for different values of $K$.



Figure 8: Temperature profiles for different values of $K$.



Figure 9: Concentration profiles for different values of $K$.

The effects of the thermal radiation parameter $R$ on velocity and temperature profiles are shown in Figures 10 and 11. It is obvious that both velocity and temperature decrease as radiation parameter $R$ increases.

The influence of the Prandtl number $\operatorname{Pr}$ on velocity and temperature field is shown in Figures 12 and 13. It is obvious that both velocity and temperature decrease as Prandtl parameter Pr increases.

Figures 14 and 15 show the dimensionless velocity and temperature profiles for different values of wall temperature parameter $\alpha$. It can be seen that both velocity and the temperature profiles decrease with the increase of wall temperature parameter $\alpha$.

Figures 16 and 17 depict the effect of Eckert number Ec on the dimensionless velocity and temperature profile. It is revealed that velocity and temperature profiles' scores grow with the increasing of the Eckert number Ec. Eckert number, physically, is a measure of frictional heat in the 


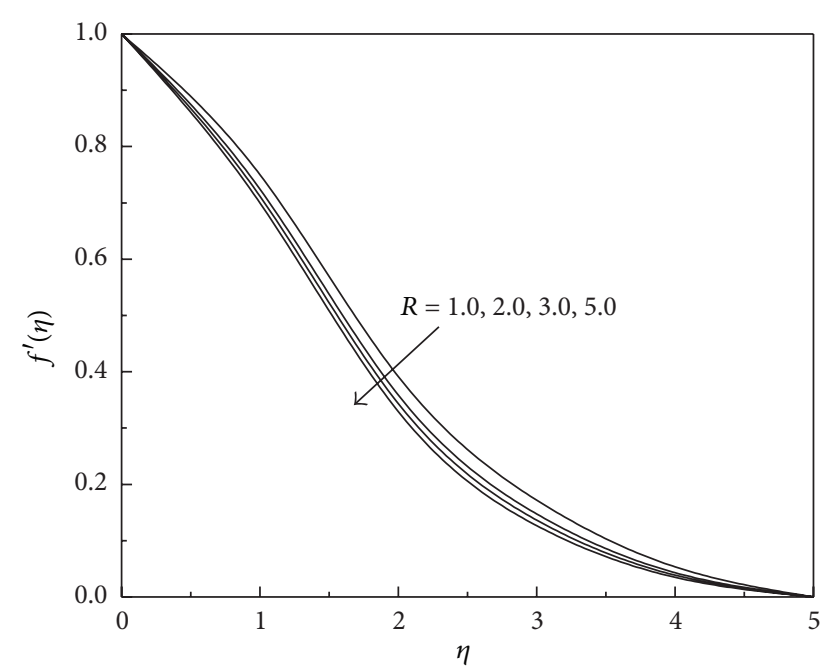

FIgURE 10: Velocity profiles for different values of $R$.

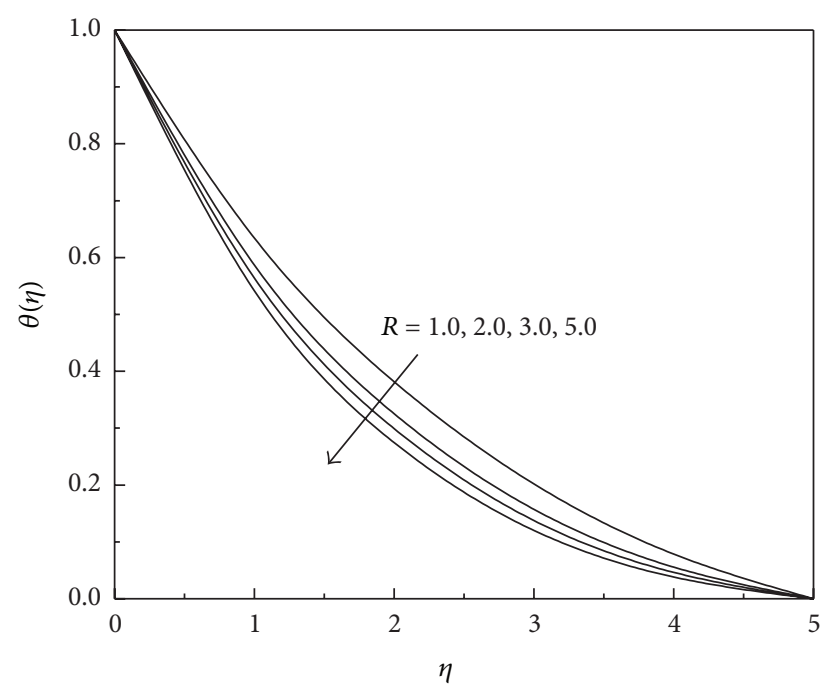

FIgURE 11: Temperature profiles for different values of $R$.

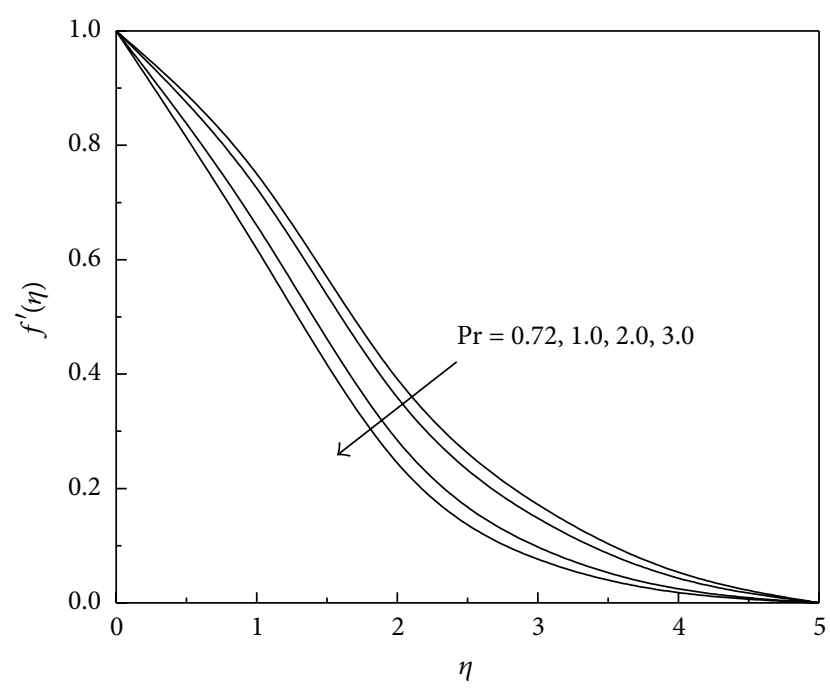

FIgURe 12: Velocity profiles for different values of Pr.

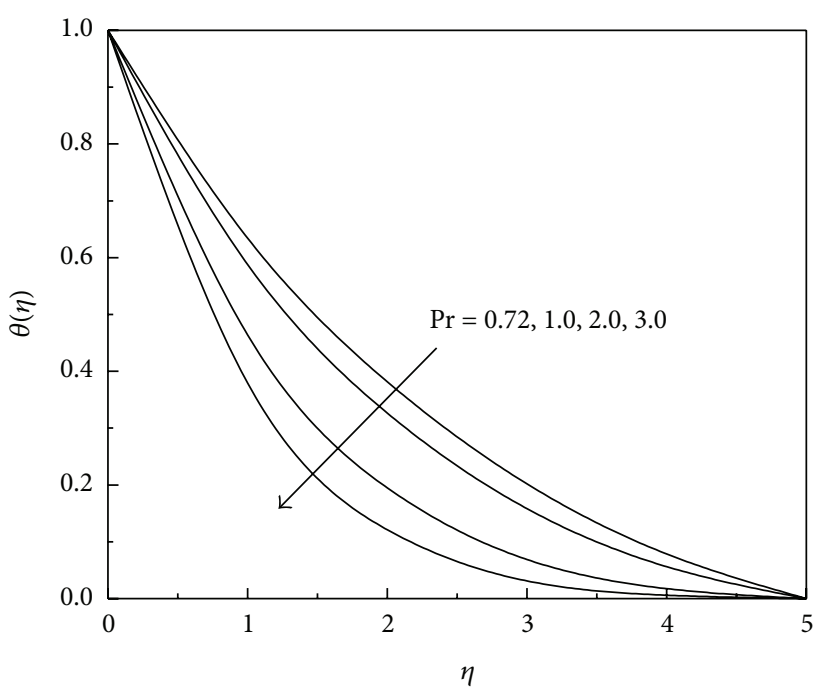

FIgURE 13: Temperature profiles for different values of Pr.

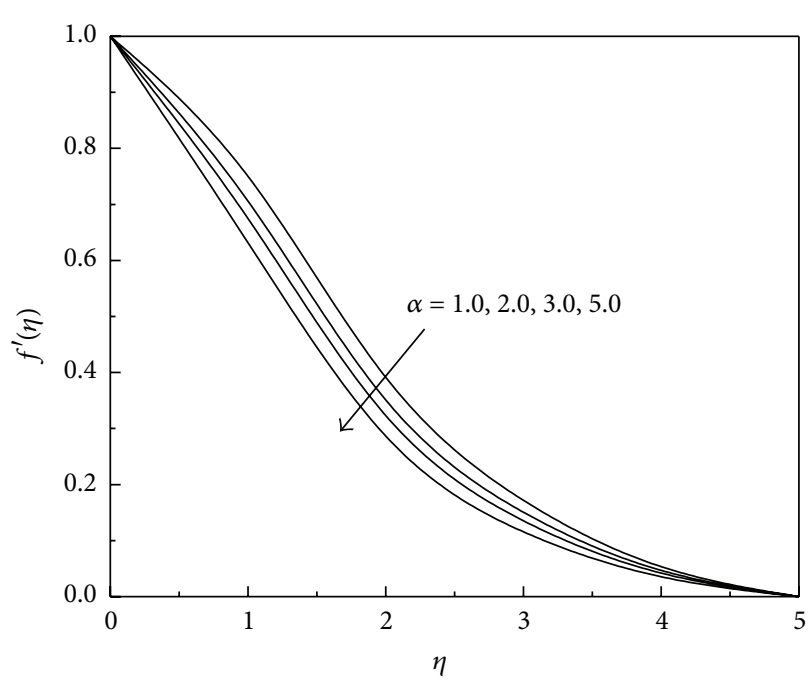

FIGURE 14: Velocity profiles for different values of $\alpha$.

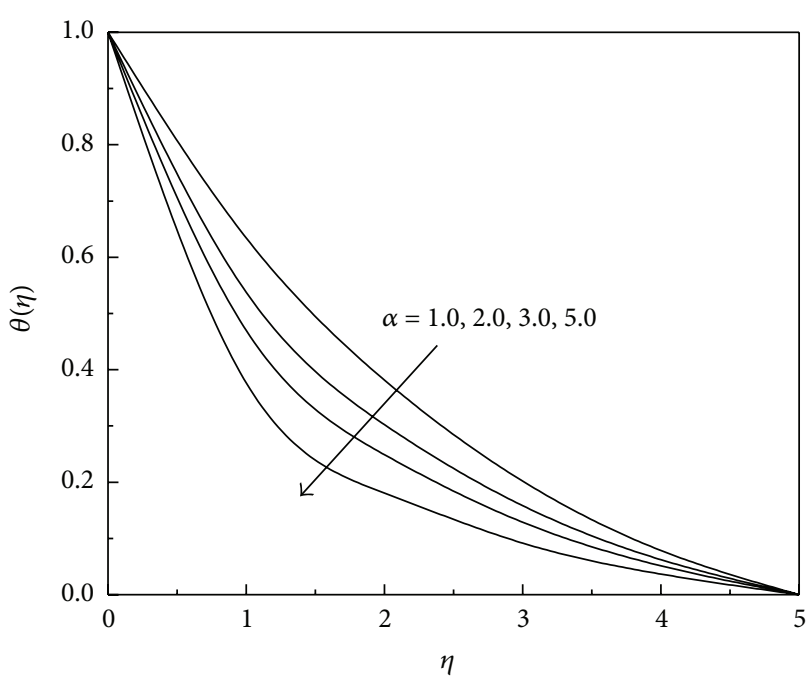

FIGURE 15: Temperature profiles for different values of $\alpha$. 




FIgURE 16: Velocity profiles for different values of Ec.

system. Hence the thermal regime with larger Ec values is subjected to rather more frictional heating causing a source of rise in the temperature. To be specific, the Eckert number Ec signifies the relative importance of viscous heating to thermal diffusion. Viscous heating may serve as energy source to modify the temperature regime qualitatively.

The influence of the Schmidt number Sc on the dimensionless velocity and concentration profiles is plotted in Figures 18 and 19. As the Schmidt number increases the concentration decreases. This causes the concentration buoyancy effects to decrease, yielding a reduction in the fluid velocity. The reductions in the velocity and concentration profiles are accompanied by simultaneous reductions in the velocity and concentration boundary layers. These behaviors are clear from Figures 18 and 19. From the definition of the Schmidt number Sc given in (9), it is clearly observed that Sc is inversely proportional to the diffusion coefficient $D$. Therefore larger values of Sc correspond to a decrease in the concentration field.

The effects of the chemical reaction parameter $\mathrm{Kr}$ on dimensionless velocity component profiles are plotted in Figure 20. As the chemical reaction parameter increases, velocity profile decreases. These behaviors are clear from Figure 20.

Figure 21 explains the variation of generative $(\mathrm{Kr}<$ 0 ) chemical reaction on the concentration field $\phi(n)$. It is noted that generative $(\mathrm{Kr}<0)$ chemical reaction causes an increase in the concentration. Figure 22 predicts the effects of destructive $(\mathrm{Kr}>0)$ chemical reaction on the concentration field $\phi(n)$. It is observed that $\phi(n)$ decreases in case of destructive $(\mathrm{Kr}>0)$ chemical reaction. It is also noticed that the magnitude of $\phi(n)$ is large in case of generative chemical reaction $(\mathrm{Kr}<0)$ in comparison to the case of destructive chemical reaction $(\mathrm{Kr}>0)$. Physically for generative case, chemical reaction $\mathrm{Kr}$ takes place without creating much disturbance whereas in the case of destructive chemical reaction is much larger. Due to this fact molecular



FIgURE 17: Temperature profiles for different values of Ec.

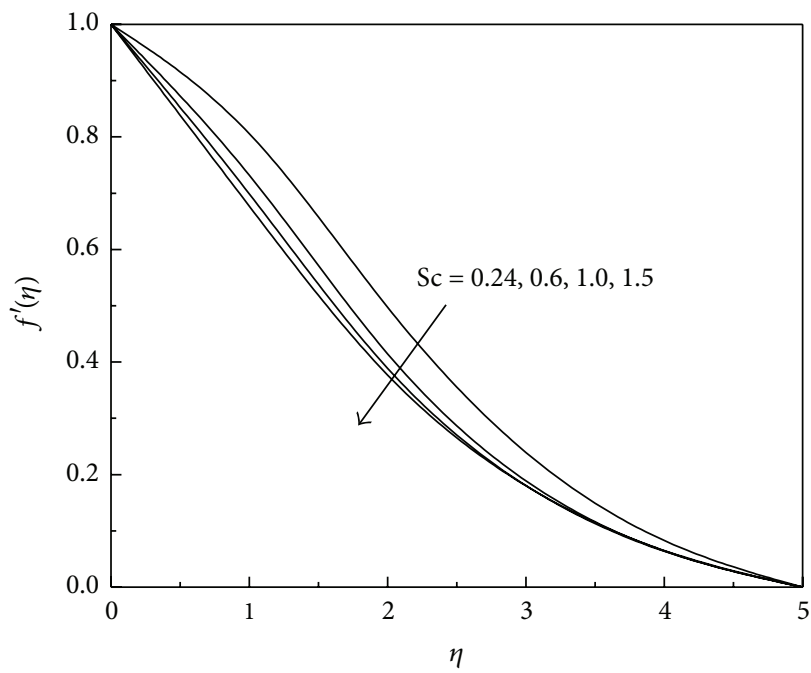

FIGURE 18: Velocity profiles for different values of Sc.



FIgURE 19: Concentration profiles for different values of Sc. 


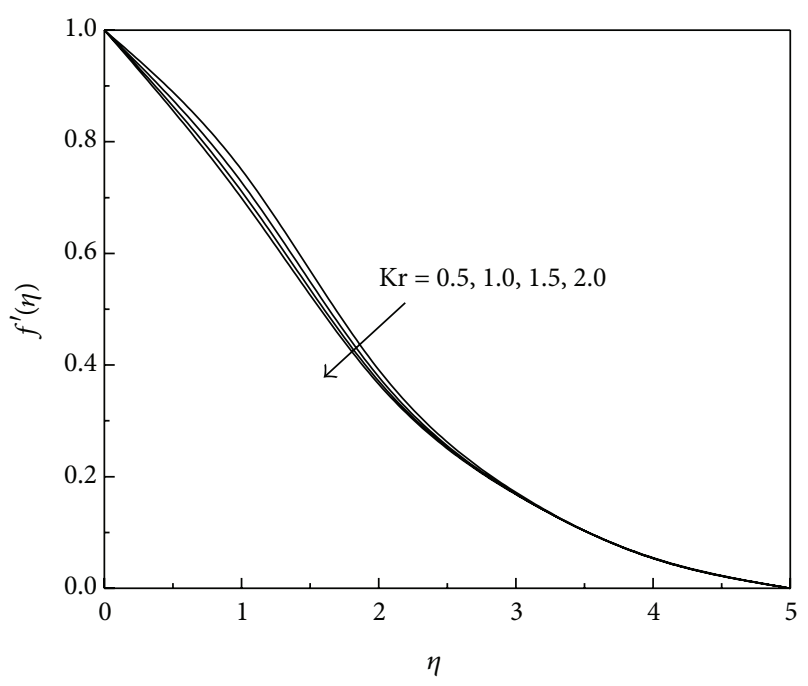

FIGURE 20: Velocity profiles for different values of Kr.



FIGURE 21: Concentration profiles for different values of $(\mathrm{Kr}>0)$.

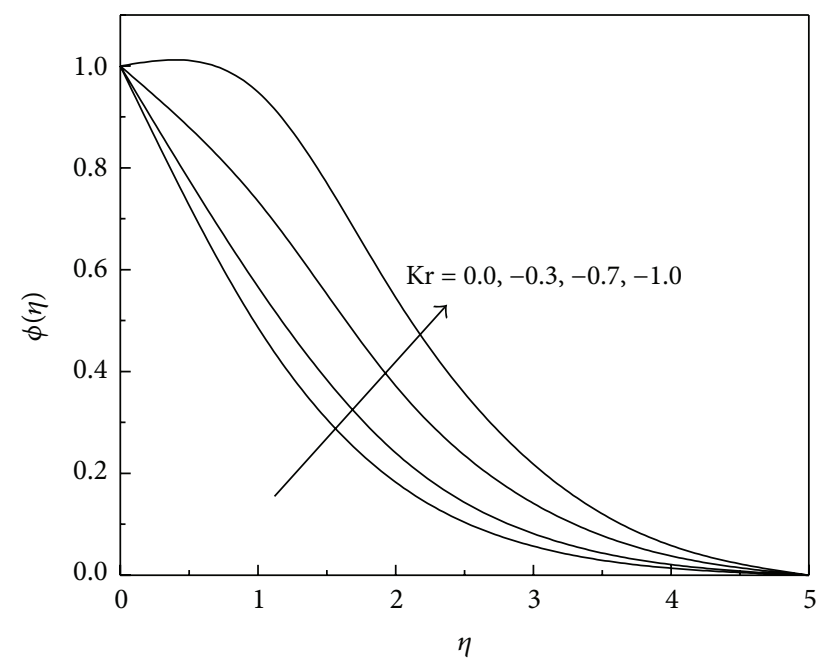

FIGURE 22: Concentration profiles for different values of $(\mathrm{Kr}<0)$.
TABLE 1: Numerical values of skin friction coefficient, Nusselt number, and Sherwood number for $\operatorname{Pr}=0.72, R=1.0, \alpha=1.0$, $\mathrm{Ec}=0.5, \mathrm{Sc}=0.6$, and $\mathrm{Kr}=0.5$.

\begin{tabular}{ccccccc}
\hline Gr & Gc & $M$ & $K$ & $f^{\prime \prime}(0)$ & $-\theta^{\prime}(0)$ & $-\phi^{\prime}(0)$ \\
\hline 2.0 & 2.0 & 1.0 & 0.5 & 0.150762 & 0.442613 & 0.777892 \\
3.0 & 2.0 & 1.0 & 0.5 & 0.553446 & 0.482143 & 0.798155 \\
4.0 & 2.0 & 1.0 & 0.5 & 0.990722 & 0.505879 & 0.816113 \\
2.0 & 3.0 & 1.0 & 0.5 & 0.497064 & 0.463111 & 0.79163 \\
2.0 & 4.0 & 1.0 & 0.5 & 0.835841 & 0.475835 & 0.804472 \\
2.0 & 2.0 & 2.0 & 0.5 & -0.270377 & 0.388834 & 0.755957 \\
2.0 & 2.0 & 3.0 & 0.5 & -0.629993 & 0.336638 & 0.738372 \\
2.0 & 2.0 & 1.0 & 1.0 & -0.068862 & 0.415729 & 0.766295 \\
2.0 & 2.0 & 1.0 & 2.0 & -0.456655 & 0.362382 & 0.746699 \\
\hline
\end{tabular}

TABLe 2: Numerical values of skin friction coefficient, Nusselt number, and Sherwood number for $\mathrm{Gr}=2.0, \mathrm{Gc}=2.0, M=1.0$, $K=1.0, \mathrm{Sc}=0.6$, and $\mathrm{Kr}=0.5$.

\begin{tabular}{lcccccc}
\hline $\operatorname{Pr}$ & $R$ & $\alpha$ & Ec & $f^{\prime \prime}(0)$ & $-\theta^{\prime}(0)$ & $-\phi^{\prime}(0)$ \\
\hline 0.72 & 1.0 & 1.0 & 0.5 & 0.150762 & 0.442613 & 0.777892 \\
1.0 & 1.0 & 1.0 & 0.5 & 0.119472 & 0.517432 & 0.77389 \\
1.5 & 1.0 & 1.0 & 0.5 & 0.0729682 & 0.635259 & 0.768052 \\
0.72 & 1.5 & 1.0 & 0.5 & 0.131292 & 0.488779 & 0.775396 \\
0.72 & 2.0 & 1.0 & 0.5 & 0.118641 & 0.519463 & 0.773784 \\
0.72 & 1.0 & 1.5 & 0.5 & 0.11505 & 0.55654 & 0.774103 \\
0.72 & 1.0 & 2.0 & 0.5 & 0.0850994 & 0.656825 & 0.770945 \\
0.72 & 1.0 & 1.0 & 0.7 & 0.178787 & 0.373412 & 0.781302 \\
0.72 & 1.0 & 1.0 & 1.0 & 0.229465 & 0.252805 & 0.787442 \\
\hline
\end{tabular}

motion in the case of $(\mathrm{Kr}>0)$ is quite larger which finally results in an increase in the mass transport phenomenon.

From Table 1, it is observed that the skin friction coefficient $f^{\prime \prime}(0)$, Nusselt number $-\theta^{\prime}(0)$, and Sherwood number $-\phi^{\prime}(0)$ decrease with the increase of magnetic field number $M$ or permeability parameter $K$. The skin friction coefficient, Nusselt number, and Sherwood number increase with the increase of Grashof number Gr or modified Grashof number Gc. From Table 2 it is found that the magnitude of the wall temperature gradient $-\theta^{\prime}(0)$ increases as Prandtl number Pr or radiation parameter $R$ or wall temperature parameter $\alpha$ increases, while it decreases as the Eckert number Ec increases. From Table 3, it is noticed that the magnitude of the wall concentration gradient $-\phi^{\prime}(0)$ decreases as the magnetic field parameter $M$ increases, while it increases with an increase in the Schmidt number Sc or chemical reaction parameter $\mathrm{Kr}$.

\section{Conclusion}

In this paper we study the chemical reaction effects on radiative MHD flow over a nonisothermal stretching sheet embedded in porous medium in the presence of viscous dissipation. The expressions for the velocity, temperature, and concentration distributions which are the equations governing the flow are numerically solved by the fourth-order Runge-Kutta method along with shooting technique. The 
TABle 3: Numerical values of skin friction coefficient, Nusselt number, and Sherwood number for $\mathrm{Gr}=2.0, \mathrm{Gc}=2.0, M=1.0$, $K=1.0, \operatorname{Pr}=0.72, R=1.0, \alpha=1.0$, and $\mathrm{Ec}=0.5$.

\begin{tabular}{lcccc}
\hline Sc & $\mathrm{Kr}$ & $f^{\prime \prime}(0)$ & $-\theta^{\prime}(0)$ & $-\phi^{\prime}(0)$ \\
\hline 0.6 & 0.5 & 0.150762 & 0.442613 & 0.777892 \\
0.78 & 0.5 & 0.116005 & 0.433268 & 0.89045 \\
1.0 & 0.5 & 0.0816344 & 0.425098 & 1.01176 \\
0.6 & 1.0 & 0.110634 & 0.433838 & 0.94824 \\
0.6 & 1.5 & 0.0794772 & 0.427467 & 1.09362 \\
\hline
\end{tabular}

effects of various governing parameters on the skin friction, Nusselt number, and Sherwood number are shown in tables. It is observed that the skin friction, Nusselt number, and Sherwood number decrease with the increase of magnetic field parameter or permeability parameter. It can be seen that the velocity profiles increase with the increase of Grashof number or modified Grashof number. It is observed that both velocity and the temperature profiles decrease with the increase of wall temperature parameter $\alpha$. As thermal radiation parameter increases, velocity and temperature profiles decrease. The concentration field $\phi(\eta)$ is a decreasing function of Schmidt number Sc. The concentration field $\phi(\eta)$ has opposite results for destructive $(\mathrm{Kr}>0)$ and generative $(\mathrm{Kr}<0)$ chemical reactions.

\section{Conflict of Interests}

The author declares that there is no conflict of interests regarding the publication of this paper.

\section{Acknowledgments}

The author would like to acknowledge the reviewers and the editor for their suggestions which led to the present form of the paper.

\section{References}

[1] M. A. Seddeek and A. A. Almushigeh, "Effects of radiation and variable viscosity on MHD free convective flow and mass transfer over a stretching sheet with chemical reaction," Applied Mathematics and Computation, vol. 5, no. 1, pp. 181-197, 2010.

[2] R. Kandasamy, T. Hayat, and S. Obaidat, "Group theory transformation for Soret and Dufour effects on free convective heat and mass transfer with thermophoresis and chemical reaction over a porous stretching surface in the presence of heat source/sink," Nuclear Engineering and Design, vol. 241, no. 6, pp. 2155-2161, 2011.

[3] D. Pal and B. Talukdar, "Combined effects of Joule heating and chemical reaction on unsteady magnetohydrodynamic mixed convection of a viscous dissipating fluid over a vertical plate in porous media with thermal radiation," Mathematical and Computer Modelling, vol. 54, no. 11-12, pp. 3016-3036, 2011.

[4] A. A. Joneidi, G. Domairry, and M. Babaelahi, "Analytical treatment of MHD free convective flow and mass transfer over a stretching sheet with chemical reaction," Journal of the Taiwan Institute of Chemical Engineers, vol. 41, no. 1, pp. 35-43, 2010.
[5] S. P. Anjalidevi and R. Kandasamy, "Effects of chemical reaction, heat and mass transfer on laminar flow along a semi infinite horizontal plate," Heat and Mass Transfer, vol. 35, no. 6, pp. 465467, 1999.

[6] M. A. Seddeek, A. A. Darwish, and M. S. Abdelmeguid, "Effects of chemical reaction and variable viscosity on hydromagnetic mixed convection heat and mass transfer for Hiemenz flow through porous media with radiation," Communications in Nonlinear Science and Numerical Simulation, vol. 12, no. 2, pp. 195-213, 2007.

[7] A. M. Salem and M. Abd El-Aziz, "Effect of Hall currents and chemical reaction on hydromagnetic flow of a stretching vertical surface with internal heat generation/absorption," Applied Mathematical Modelling, vol. 32, no. 7, pp. 1236-1254, 2008.

[8] D. A. Nield and A. Bejan, Convection in Porous Media, Springer, New York, NY, USA, 2nd edition, 1999.

[9] M. S. Abel, S. K. Khan, and K. V. Prasad, "Convective heat and mass transfer in a visco-elastic fluid flow through a porous medium over a stretching sheet," International Journal of Numerical Methods for Heat and Fluid Flow, vol. 11, no. 8, pp. 779-792, 2001.

[10] P. Vyas and N. Srivastava, "Radiative MHD flow over a nonisothermal stretching sheet in a porous medium," Applied Mathematical Sciences, vol. 4, no. 49-52, pp. 2475-2484, 2010.

[11] K. Vajravelu and A. Hadjinicolaou, "Convective heat transfer in an electrically conducting fluid at a stretching surface with uniform free stream," International Journal of Engineering Science, vol. 35, no. 13-12, pp. 1237-1244, 1997.

[12] S. Mohammed Ibrahim and N. Bhaskar Reddy, "Similarity solution of heat and mass transfer for natural convection over a moving vertical plate with internal heat generation and a convective boundary condition in the presence of thermal radiation, viscous dissipation and chemical reaction," ISRN Thermodynamics, vol. 2013, Article ID 790604, 10 pages, 2013.

[13] S. Shateyi, P. Sibanda, and S. S. Motsa, "Magnetohydrodynamic flow past a vertical plate with radiative heat transfer," Journal of Heat Transfer, vol. 129, pp. 1708-1714, 2007.

[14] O. D. Makinde and P. Sibanda, "Magnetohydrodynamic mixedconvective flow and heat and mass transfer past a vertical plate in a porous medium with constant wall suction," Journal of Heat Transfer, vol. 130, no. 11, pp. 1-8, 2008.

[15] D. Pal and B. Talukdar, "Perturbation analysis of unsteady magnetohydrodynamic convective heat and mass transfer in a boundary layer slip flow past a vertical permeable plate with thermal radiation and chemical reaction," Communications in Nonlinear Science and Numerical Simulation, vol. 15, no. 7, pp. 1813-1830, 2010.

[16] O. D. Makinde and A. Aziz, "MHD mixed convection from a vertical plate embedded in a porous medium with a convective boundary condition," International Journal of Thermal Sciences, vol. 49, no. 9, pp. 1813-1820, 2010.

[17] R. Cortell, "Effects of viscous dissipation and radiation on the thermal boundary layer over a nonlinearly stretching sheet," Physics Letters A: General, Atomic and Solid State Physics, vol. 372, no. 5, pp. 631-636, 2008.

[18] F. S. Ibrahim, A. M. Elaiw, and A. A. Bakr, "Effect of the chemical reaction and radiation absorption on the unsteady MHD free convection flow past a semi infinite vertical permeable moving plate with heat source and suction," Communications in Nonlinear Science and Numerical Simulation, vol. 13, no. 6, pp. 1056-1066, 2008. 
[19] S. Shateyi, "Thermal radiation and buoyancy effects on heat and mass transfer over a semi-infinite stretching surface with suction and blowing," Journal of Applied Mathematics, vol. 2008, Article ID 414830, 12 pages, 2008.

[20] S. Shateyi and S. S. Motsa, "Thermal radiation effects on heat and mass transfer over an unsteady stretching surface," Mathematical Problems in Engineering, vol. 2009, Article ID 965603, 13 pages, 2009.

[21] V. Aliakbar, A. Alizadeh-Pahlavan, and K. Sadeghy, "The influence of thermal radiation on MHD flow of Maxwellian fluids above stretching sheets," Communications in Nonlinear Science and Numerical Simulation, vol. 14, no. 3, pp. 779-794, 2009.

[22] B. Gebhart, "Effects of viscous dissipation in natural convection," Journal of Fluid Mechanics, vol. 14, pp. 225-232, 1962.

[23] P. Vyas and N. Srivastava, "On dissipative radiative MHD boundary layer flow in a porous medium over a non-isothermal stretching sheet," Journal of Applied Fluid Mechanics, vol. 5, no. 4, pp. 23-31, 2012.

[24] B. Gebhart and J. Mollendorf, "Viscous dissipation in external natural convection flows," Journal of Fluid Mechanics, vol. 38, no. 1, pp. 97-107, 1969.

[25] D. A. Nield, "Resolution of a Paradox involving viscous dissipation and nonlinear drag in a porous medium," Transport in Porous Media, vol. 41, no. 3, pp. 349-357, 2000.

[26] D. A. S. Rees, E. Magyari, and B. Keller, "The development of the asymptotic viscous dissipation profile in a vertical free convective boundary layer flow in a porous medium," Transport in Porous Media, vol. 53, no. 3, pp. 347-355, 2003.

[27] M. Q. Brewster, Thermal Radiation Transferproperties, John Wiley \& Sons, New York, NY, USA, 1972.

[28] A. J. Chamkha, "Hydromagnetic natural convection from an isothermal inclined surface adjacent to a thermally stratified porous medium," International Journal of Engineering Science, vol. 35, no. 10-11, pp. 975-986, 1997. 




Advances in

Operations Research

mansans



The Scientific World Journal
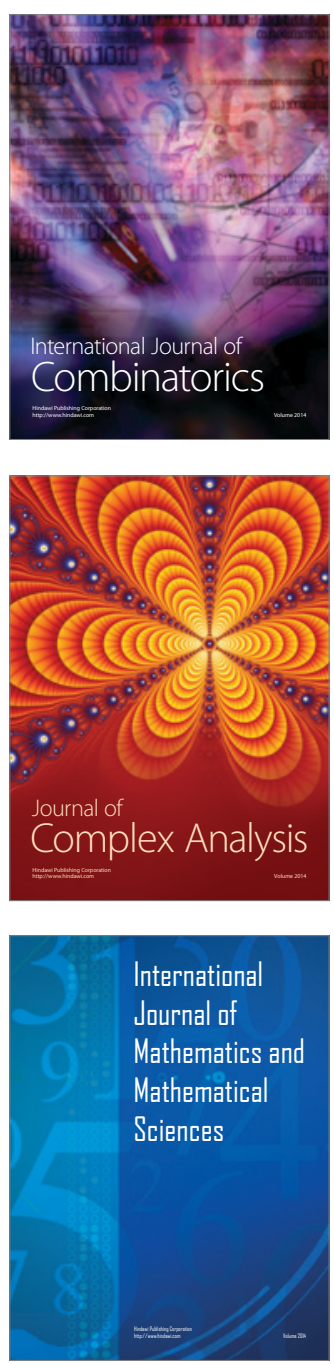


Submit your manuscripts at http://www.hindawi.com
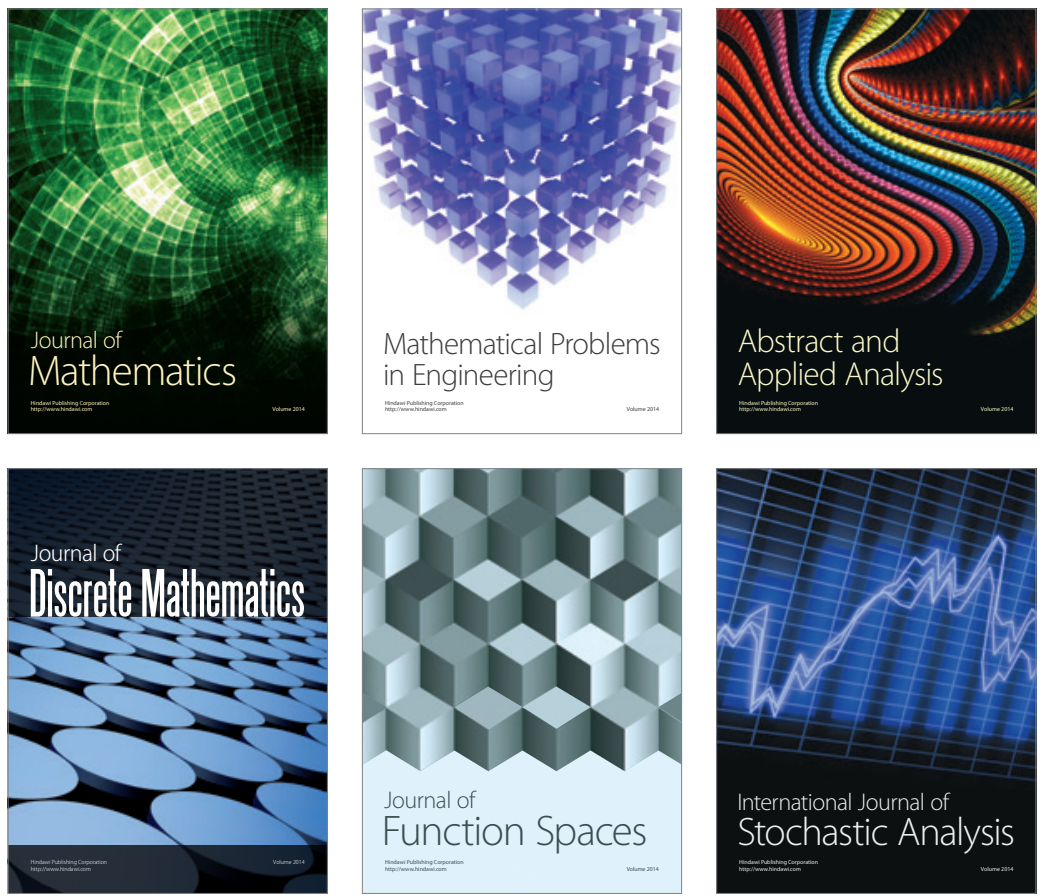

Journal of

Function Spaces

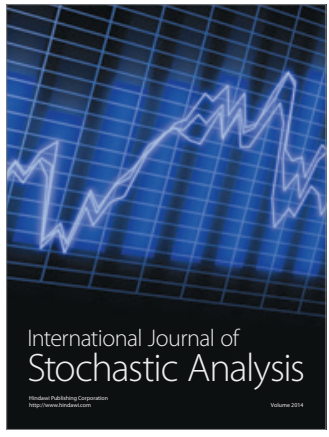

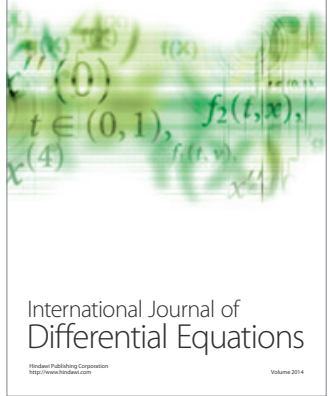
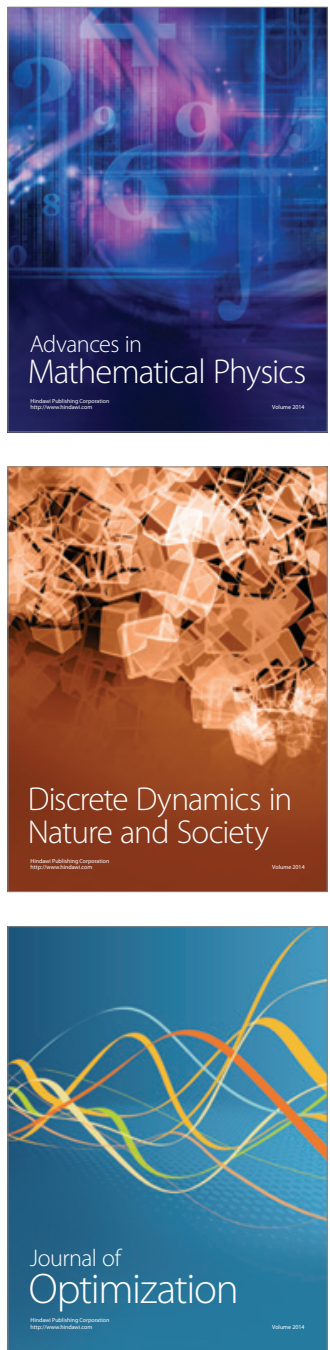